\title{
Minority report
}

\section{If American science is to meet the needs of all of its citizens, its scientists must reflect the diversity of the country's population.}

$\mathbf{T}$ he typical American lab is peopled almost entirely with white scientists. That's not reflective of society at large. A shake-up of the way minorities are recruited, trained and promoted could give minority representation in science the boost it so badly needs.

In 2000 , the US population was $75 \%$ white, $12 \%$ black and $12 \%$ Hispanic. But the proportion of minorities that completed biology PhDs between 1993 and 2002 did not match these numbers: only $2.6 \%$ of new PhDs were black and only $3.7 \%$ were Hispanic. The proportion of tenure-track biology faculty in 2002 was even more disparate: $89 \%$ white, $1 \%$ black and $2 \%$ Hispanic.

These disturbing statistics tell only part of the story. According to first-person accounts, because minorities are often the only one of their ethnicity in their lab or department-perhaps even in their institution-they often feel isolated from their coworkers. Because they lack colleagues from their own ethnic group, they may feel unable to effect institutional changes to address the unique challenges they face.

It is clear that change is necessary. According to the Commission on Professionals in Science and Technology, in 2005 salaries for minority biology researchers were as low as $80 \%$ of the salaries of their non-minority counterparts. Only when there is full disclosure of all academic salaries will this inequality begin to disappear.

Correcting salary disparities is only the first step. Funding agencies, professional societies and universities must work harder to attract and retain minority scientists. They must ensure that minorities have committed mentors to help them through the intricacies of grant applications, job interviews and salary negotiations. Professional societies should make every effort to enhance the participation of minority professors so that their visibility motivates the next generation of scientists.

Minorities can also find inspiration in the changing status of women scientists, whose presence in academia has risen steadily over the last 20 years. Programs that are intended to assist female scientists, such as the ADVANCE fellowship from the US National Science Foundation (NSF), have helped retain women who otherwise might have left science. Programs tailored for minorities may be similarly effective in attracting and retaining them.

Minority-targeted funding is available at all levels of the academic ladder. Some universities provide internal funding for minority student research. The US National Institutes of Health award minority fellowships: for example, the National Institute on Aging plans to award $\$ 250,000-300,000$ per year for up to 8 minority doctoral fellowships. The NSF has committed $\$ 3$ million for 36 minority postdoctoral fellowships and 6 'starter' grants to initiate minority-led independent research programs. But this funding will only be in place if the money remains available in the NSF budget. Scientists must lobby Congress so that funding for these programs is not just maintained, but increased.

Are these fellowships enough? Have minority-targeted recruitment efforts been effective at attracting underrepresented groups? According to a study by the US National Academies' Research Council, the outcome of these programs is unclear because they lack a built-in method for assessing their effectiveness.

However, anecdotal evidence from the study warns that these programs are falling short of their goal. For example, minority postdoctoral trainees are more likely to embark on another postdoctoral position, while non-minorities are more likely to apply for independent academic professorships. And many more undergraduate minorities in scientific training programs reported negative experiences with their mentor, with some being given administrative tasks instead of lab work. Universities must correct these inequalities so that underrepresented groups are treated more fairly during their training.

Many resources can empower underrepresented groups, and universities should ensure their trainees utilize these resources. For example, professional societies such as the NIH Black Scientists Association give minorities a voice and provide mentoring to scientists of all levels. If minority scientists do not have role models at their local institution, Internet communities such as justgarciahill.org, which serves all underrepresented groups, can provide inspiration and guidance.

Minority representation in science will not increase overnight. But the need for diversity at the bench and in the highest echelons of science cannot wait another generation. Investments must be made now to recruit and retain minority scientists so that their representation increases to reflect the importance of diverse voices in scientific research. 